Annuaire suisse de politique de développement

16 | 1997

Environnement et développement, 5 ans après Rio

\title{
Commerce, importation et exportation de biens culturels : état de la réglementation en Suisse
}

Catherine Schümperli Younossian

\section{OpenEdition}

1 Journals

Édition électronique

URL : http://journals.openedition.org/aspd/819

DOI : 10.4000/aspd.819

ISSN : 1663-9669

Éditeur

Institut de hautes études internationales et du développement

Édition imprimée

Date de publication : 1 mars 1997

Pagination : 271-282

ISSN : 1660-5934

\section{Référence électronique}

Catherine Schümperli Younossian, «Commerce, importation et exportation de biens culturels : état de la réglementation en Suisse ", Annuaire suisse de politique de développement [En ligne], 16 | 1997, mis en ligne le 09 août 2012, consulté le 08 septembre 2020. URL : http://journals.openedition.org/aspd/ 819 ; DOI : https://doi.org/10.4000/aspd.819 


\title{
COMMERCE, IMPORTATION ET EXPORTATION DE BIENS CULTURELS : ÉTAT DE LA RÉGLEMENTATION EN SUISSE
}

CATHERINE SCHÜMPERLI YOUNOSSIAN

\begin{abstract}
A u cours des dernières décennies, le commerce international des biens culturels de toute nature (peintures, sculptures, antiquités, objets archéologiques, art moderne, etc.) s'est considérablement développé, voire emballé. Jusque dans les années 60 , le marché de l'art était surtout l'affaire de collectionneurs esthètes. Mais déjà apparaissaient les premiers acheteurs-spéculateurs, qui cherchaient à faire de bons placements. Désormais, les biens culturels sont de plus en plus considérés comme des marchandises. Dans les années $80 / 90$, la prospérité a favorisé le développement du marché de l'art. A titre d'exemple, l'une des plus importantes maisons de ventes aux enchères - Sotheby's - a affiché un chiffre d'affaires de 382 millions de dollars pour l'exercice $1979 / 80$ et de 3,2 milliards de dollars en 1989/90, soit une augmentation de $800 \%$ en dix ans. L'augmentation du chiffre d'affaires du commerce de l'art est également due à la croissance du commerce illicite de biens culturels. Les bénéfices qu'il occasionne permettent le blanchiment de l'argent de mafias diverses, des bénéfices de la drogue et d'autres trafics. C'est précisément sur les abus du commerce illégal de biens culturels et sur les propositions politiques et juridiques destinées à faire face à cette situation que cet article tente d'apporter des éléments d'analyse.'
\end{abstract}

\section{MÉMOIRE OU BIENS MARCHANDS}

La question du commerce de l'art est d'autant plus délicate que les objets d'art et de culture ne peuvent pas être entièrement assimilés aux autres biens commerciaux ; leur valeur historique, leur fonction de témoin d'une identité culturelle, leur caractère unique et non reproductible, ainsi que d'autres facteurs qui échappent à l'analyse rationnelle les en distinguent. Ces particularités, jointes à la valeur immatérielle des biens culturels, ont conduit de nombreux Etats à interdire l'exportation de ces objets, qu'ils considèrent appartenir à leur patrimoine culturel. ${ }^{2}$ Comme le souligne le rapport d'un groupe de travail ${ }^{3}$, l'attrait pour les objets archéologiques n'est pas sans conséquence : « l'augmentation des prix des biens culturels conduit toujours davantage au pillage des pays méditerranéens, riches en biens culturels, mais aussi de certains pays d'Asie, d'Amérique centrale et d'Afrique, par le crime organisé. Il ne s'agit pas là de cas isolés de vols

\footnotetext{
Afin de comprendre la chronologie des faits marquants, le lecteur pourra se référer à un encadré à la fin de l'article.

${ }^{2}$ Commerce, importation et exportation de biens culturels, Texte et rapport explicatif du Département fédéral de l'intérieur, Berne, août 1993.

3 En janvier 1990, l'office fédéral de la culture a institué un groupe de travail afin d'étudier toutes les questions se rapportant au commerce de biens culturels. Le rapport le commerce, l'exportation et l'importation de biens culturels en Suisse. Portée nationale et internationale; problèmes, solutions et effets lui a été remis en janvier 1991. Citation page 8 du rapport.
} 
d'œuvres d'art qui rencontrent un écho dans le monde entier, mais bien de fouilles clandestines systématiques effectuées sur une grande échelle. Ceux qui détruisent des sites archéologiques font disparaître d'importantes sources de renseignements historiques, qui sont perdues à tout jamais pour la recherche scientifique. » Depuis la chute du mur de Berlin ce sont les pays d'Europe de l'Est qui doivent faire face au pillage systématique de leur patrimoine culturel. Les musées de Roumanie ont perdu 11'000 œuvres en trois ans. Ils ont été victimes de 60 cambriolages, parfois à main armée. Les autorités russes ont établi l'existence de 40 réseaux organisés impliqués dans le trafic des trésors artistiques de leur pays. En 1992, on évaluait à 20'000 le nombre d'objets sortis en contrebande de la République tchèque. Plus de 1'100 églises ont été dévalisées en Pologne soit une augmentation de plus de $100 \%$ depuis le début des années $80 .{ }^{4}$ Le Sud n'est pas épargné, de nombreux pays d'Asie, d'Afrique et d'Amérique latine ont été affectés par des pillages de biens culturels et victimes de commerce illicite. Le Mali, par exemple, a été fortement touché, comme en témoigne Samuel Sidibé, archéologue et directeur du Musée national malien à Bamako, qui affirme que ces vingt dernières années une grande partie de l'héritage culturel malien est partie en direction de l'Europe et des Etats-Unis. Toutes les régions du Mali ont été victimes de pillages systématiques. Dans certaines régions, 80 à $90 \%$ des sites archéologiques ont été pillés. ${ }^{5}$ Cette situation a amené les Etats-Unis à conclure avec le Mali (ainsi qu'avec le Mexique, le Pérou et le Guatemala, pays spécialement affectés par cette situation) des accords bilatéraux sur la restitution de biens culturels volés. Selon la célèbre revue " Archaeology », certains experts estiment que plus de $90 \%$ des objets archéologiques qui se trouvent sur le marché aujourd'hui proviennent de fouilles illégales. ${ }^{6}$

Comme le souligne Pierre Lalive d'Epinay ${ }^{7}$ dans un article publié à propos de la Convention d'Unidroit « le conflit d'intérêts entre pays "exportateurs", souvent involontaires, de biens culturels et pays "importateurs", a pris à l'évidence d'inquiétantes proportions, susceptibles de menacer l'entente et la paix internationales. Certes, il ne faut pas être dupe de schémas simplificateurs comme celui de l'opposition Nord-Sud, riches-pauvres, exportateurs-importateurs, etc. Bien des pays sont déjà (dont le meilleur exemple est sans doute l'Italie) à la fois dans la situation d'exportateurs et d'importateurs de biens d'art ou autres objets culturels, et il est souhaitable qu'ils le soient ou le deviennent dans l'intérêt des échanges culturels internationaux (...). Il n'en reste pas moins que d'importantes différences de fait existent à l'heure actuelle et sont fortement ressenties (...)». Cette affirmation est partiellement remise en cause par les organisations de développement qui constatent que le flux de biens culturels entre les pays du tiers monde et les pays industrialisés est, de fait, à sens unique.

\footnotetext{
INFO, Fiche d'information de la Commission nationale suisse pour l'UNESCO, août 1993. in Illicit Traffic in Cultural Property, Samuel Sibidé, Ed. by Harrie Leyten, Amsterdam 1995, p. 28.

in Archaeology, sept./oct. 1992, p. 67.

in « Une avancée du droit international : la Convention de Rome d'Unidroit sur les biens culturels volés ou illicitement exportés ", Revue de droit uniforme 1996-1, p. 57.
} 


\section{LE RÔLE DE LA SUISSE}

La Suisse occupe une place très importante sur le marché de l'art puisqu'elle occupe la quatrième position au niveau mondial après la Grande-Bretagne, les USA et la France. En 1995, elle a importé des « objets d'art, de collection ou d'antiquité » pour une valeur de 315 millions de dollars et en a exporté pour 415 millions de dollars. ${ }^{8}$ Ces chiffres correspondent aux données officielles, et ne tiennent pas compte des importations illégales. En 1992, le magazine Bilanz ${ }^{9}$ consacrait un article au commerce illégal de l'art et à ses bénéfices. Il mentionnait quelques estimations du chiffre d'affaires du commerce illégal de l'art au niveau mondial qui, selon les sources, varie de 1,3 milliard de francs suisses (London Art Loss Register), 2,6 milliards de francs (selon des estimations du gouvernement américain) à 9 milliards de francs (selon le magazine britannique Trace). Le marché de l'art est très peu transparent. Il existe un commerce tout à fait licite, indispensable à la circulation des biens dans les musées publics et les collections privées, en revanche le commerce illicite est inadmissible. Ces dernières années un nombre important d'abus ont été dénoncés, les trafiquants sachant parfaitement utiliser les faiblesses de la législation suisse.

\section{LACUNE DU DROIT SUISSE}

L'attrait de la Suisse provient entre autres du laxisme extrême de sa législation. On ne dispose pas actuellement sur le plan fédéral de normes légales en matière de contrôle de l'exportation de biens culturels. S'agissant de leur importation, elle est libre en Suisse. De plus, aucun canton n'a édicté de prescriptions concernant le contrôle de l'importation de biens culturels, ce qui s'explique aisément dès lors que les cantons ne sont pas compétents en matière douanière. ${ }^{10}$

D'autre part, en droit suisse (code civil) les biens culturels sont malheureusement considérés comme une marchandise pareille aux autres. Il n'existe aucune disposition particulière d'ordre douanier concernant leur importation et leur exportation. En outre, la législation suisse protège mieux l'acquéreur de bonne foi que les victimes du vol. L'acquéreur bona fide doit être dédommagé au cas où l'objet est restitué, même s'il est établi que l'objet a été volé. Le délai pour une demande de restitution est seulement de 5 ans, passé ce délai, un acquéreur de bonne foi ne peut plus être poursuivi en justice. Les lois fiscales suisses préservent l'anonymat du vendeur et de l'acheteur et les activités de vente aux enchères profitent de ces lois permissives. De plus l'entreposage et le commerce de biens culturels sont possibles dans les ports francs sans contrôle importun. Les marchandises doivent être munies d'un simple document de transit sur lequel l'indication, objets de bois par exemple, suffit.

La Suisse est cependant bien armée avec la loi fédérale sur l'entraide pénale, qui permet à tous les pays pillés de faire une demande pour obtenir la saisie et la restitution de biens culturels volés. Cette loi exige une double incrimination, c'està-dire que les faits exposés dans la demande d'entraide doivent correspondre aux

${ }^{8}$ Statistiques du commerce extérieur de la Suisse, 1995.

9 in Bilanz 6/1992, "Geklaut wird auf Bestellung ", p. 49.

${ }^{10}$ Rapport final de la journée d'information Biens culturels : mémoires ou marchandises ?, Commission nationale suisse pour l'UNESCO, Berne, 1993, p. 42-44. 
éléments constitutifs d'une infraction réprimée en droit suisse. On comprend mieux la difficulté d'application de cette loi quand on sait qu'il n'est pas toujours facile de prouver le vol d'objets archéologiques issus de fouilles clandestines. Il faut cependant rappeler le nombre croissant de demandes d'entraide judiciaire adressées à la Suisse dans des affaires pénales de vol ou d'exportation illégale de biens culturels, ainsi que les décisions judiciaires demandant la restitution au pays d'origine d'objets ayant transité par la Suisse.

La Suisse ne connaît donc ni prescriptions, ni contrôles de l'importation et de l'exportation de biens culturels, contrairement aux pays qui l'entourent. Constatant ce vide juridique, le groupe de travail écrit dans son rapport « il est évident que notre pays est devenu une plaque tournante internationale pour le transit et le commerce d'œuvres d'art et de biens culturels d'origine illégale. $"{ }^{11}$ Cet attrait devrait encore s'accroître du fait que les pays de l'UE et de l'EEE ont adopté une directive de « Restitution » et un règlement d' " Exportation » des biens culturels. De plus, les Etats-Unis et le Canada, ainsi que 80 autres Etats, ont ratifié la Convention de l'UNESCO de 1970, qui vise à empêcher le commerce illicite de biens culturels. Ridha Fraoua, de l'Office fédéral de Justice, déclarait dans une interview parue en juillet 1995 dans un journal romand ${ }^{12}:$ «Pour les négociants et les collectionneurs privés, le territoire suisse devient ainsi plus intéressant pour faire des affaires illégales ». Et de compléter, « il ne fait aucun doute que la situation juridique actuelle en Suisse est propice à une augmentation des abus. La discussion politique à venir sur ce thème "chargé d'émotion" est donc bienvenue. Il n'existe aucune vue d'ensemble sur le nombre des vols d'œuvre d'art en Suisse car la protection des biens culturels est l'affaire des cantons. On sait toutefois qu'il est très largement supérieur au nombre de procès. "

Dans la conclusion du rapport du groupe de travail (janvier 1991) deux propositions étaient faites pour pallier à cette situation :

- la création d' une base constitutionnelle, inexistante à ce jour, pour permettre à la Confédération d'intervenir pour lutter efficacement contre le trafic illicite de biens culturels. Dans un deuxième temps, une loi fédérale d'application devrait être élaborée.

- la ratification de la Convention du l'UNESCO de 1970 concernant les mesures à prendre pour interdire et empêcher l' importation, l' exportation et le transfert de propriété illicites de biens culturels.

Depuis lors, la situation du droit international a évolué puisqu'une nouvelle convention a été signée en 1995 à Rome, la Convention d'Unidroit du 24 juin 1995 sur le retour international des biens culturels volés ou illicitement exportés. Se disant "préoccupé par la multiplication inquiétante des pratiques illégales observées dans le domaine de la circulation internationale de biens culturels $»^{13}$, le Conseil fédéral a lancé deux procédures de consultation auprès des milieux intéressés. La première, lancée en septembre 1993, se rapportait aux deux propositions du groupe de travail (voir ci-dessus). La seconde, qui a débuté en janvier 1996, visait à prendre l'avis des milieux concernés sur la ratification de la Convention d'Unidroit.

${ }^{11}$ Le commerce, l'exportation et l'importation de biens culturels en Suisse. Portée nationale et internationale ; problèmes, solutions et effets. Rapport du groupe de travail à l'intention de l'Office fédéral de la culture et du Département fédéral de l'intérieur, janvier 1991, p.10.

${ }^{12}$ La Liberté, 18 juillet 1995, «La restitution des œuvres d'art volées sera réglementée ».

${ }^{13}$ Lettre du 29 février 1996 de Ruth Dreifuss, accompagnant la procédure de consultation de la Convention d'Unidroit. 
En 1960 le Pérou et le Mexique demandaient, dans le cadre de l'UNESCO, que des mesures soient prises pour lutter contre le commerce illicite d'objets d'art. En 1964 un comité d'experts était institué pour préparer un avant-projet de convention internationale. Cet avant-projet était présenté en août 1969, et le 14 novembre 1970, la Convention de l'UNESCO était signée.

La Convention exige la restitution de matériel artistique volé et exporté illégalement. Cette décision n'a pourtant pas d'effet rétroactif et englobe uniquement les objets provenant de musées et d'institutions internationales. Les Etats signataires s'engagent en outre à contrôler l'exportation et l'importation de biens culturels, à collaborer dans l'établissement d'inventaires et à s'informer du traitement de ces objets ; ils s'engagent également à se soutenir mutuellement lors de découverte de transactions illégales. Quatre vingt-deux Etats, parmi lesquels de nombreux pays européens, les Etats-Unis et le Canada ont déjà ratifié la Convention de l'UNESCO. Etant d'application indirecte (non self-executing), les dispositions de la Convention doivent d'abord être formellement intégrées dans la législation de chaque Etat contractant. Un certain nombre de critiques ont été émises à l'encontre de la Convention. Sa formulation, très générale, laisse une importante liberté dans sa transposition dans le droit national, en particulier pour ce qui est de la définition des biens culturels. Certains reprochent au texte des tendances au nationalisme culturel et des préoccupations trop unilatérales; d'où son peu de succès auprès des Etats « importateurs » de biens culturels. De plus la Convention se caractérise par un champ d'application extrêmement limité, puisqu'elle ne traite que de la restitution des biens culturels volés dans les musées ou les institutions analogues. Consciente de ses limites, l'UNESCO a du reste demandé à Unidroit d'étudier cette question.

En septembre 1993, le Conseil fédéral a ouvert une procédure de consultation auprès des milieux intéressés concernant l'opportunité de la ratification de la Convention de l'UNESCO. Les résultats de la consultation, rendus publics en janvier 1996, laissent apparaître qu'une écrasante majorité des organisations consultées $(75 \%)$ souhaite donner à la Confédération compétence pour légiférer dans le domaine du commerce international de biens culturels, approuve la ratification de la Convention de l'UNESCO et est favorable à l'élaboration d'une loi fédérale. Les deux pôles du débat sont constitués par les acteurs du marché de l'art, farouchement opposés à toute réglementation, d'une part, et les organisations de développement d'autre part. Les premiers contestent jusqu'à l'existence d'un danger de pillage des ressources culturelles des pays pauvres : les échanges culturels sont pour eux indispensables et profitent à tous. Les seconds estiment au contraire impératif de prendre des mesures contre les abus et le trafic illégal. Dans ce contexte, et malgré ses faiblesses, une ratification de la Convention serait un signe politique, qui témoignerait de la détermination de la Suisse à coopérer dans la lutte contre toute activité illicite. Autour de ces deux pôles, la majorité de partisans de la réglementation regroupe la plupart des associations culturelles, ainsi que toutes les associations suisses des musées, les partis, les cantons et les organisations diverses (associations de développement, féminines et ecclésiastiques). Seuls sont opposés, en plus des organisations de commerçants d'art, le Vorort et l'Union suisse des arts et métiers (Usam), les cantons de Genève et Vaud, l'UDC et le PLS. Selon le dossier de presse du Département de 
l'intérieur, rendu public en janvier 1996, « les résultats de la consultation confortent le Conseil fédéral dans son intention de légiférer dans ce domaine, un besoin de réglementation se faisant sentir pour des raisons tant de politique culturelle que de politique étrangère $» .^{14}$

\section{LA CONVENTION D'UNIDROIT}

Le texte de la Convention d' Unidroit du 24 juin 1995 sur le retour international des biens culturels volés ou illicitement exportés est le résultat de dix années de travaux, auxquels ont participé deux organisations, l'UNESCO et l'Institut Unidroit (l'Institut international pour l'unification du droit privé), ainsi qu'un grand nombre d'experts. La Suisse était associée aux travaux dès le début; dans les commissions et lors de la conférence diplomatique, qui s'est tenue du 7 au 24 juin 1994 à Rome, elle a marqué de son empreinte les débats et a su jouer un rôle d'intermédiaire important. Septante-huit Etats représentant tous les continents ont participé à la conférence. Jusqu'à présent vingt-deux pays, dont l'Italie, la France, la Finlande, la Hollande et la Suisse, ont signé la Convention.

\section{Exemple de demande de restitution par des Etats Vente aux enchères d'art précolombien}

Le 7 novembre 1995, la Galerie Koller à Zurich a vendu aux enchères plus de 400 pièces d'art précolombien du Pérou, de Bolivie, du Mexique et du Guatemala. La plus grande partie de ces pièces provenaient du Pérou.

L'Ambassadeur du Pérou avait été mis au courant de cette vente aux enchères et s'était adressé aux personnes compétentes au Pérou afin de savoir si ces pièces avaient été exportées légalement. Les investigations entreprises démontrèrent que les biens culturels en question provenaient de fouilles illégales. Au Pérou, en effet, tous les biens culturels appartenant soit à des musées, soit à des privés doivent être enregistrés. Les pièces mises en vente à Zurich ne l'étaient pas et de l'avis du Ministère péru- vien de la culture elles avaient été déterrées de manière illégale. Durant ces vingt dernières années le Pérou n'avait accordé aucune autorisation d'exportation pour des pièces similaires. Par la suite, le Pérou a tenté de stopper la vente aux enchères de la Galerie Koller et a rédigé une note diplomatique de protestation à l'attention du Département des affaires étrangères. La vente n'a cependant pas pu être empêchée, la raison étant une fois de plus les lacunes juridiques en matière de vente de biens culturels de provenance illégale. Par la suite, le Pérou a fait appel à Interpol et une enquête suit son cours en collaboration avec la police du canton de Zurich. II est peu vraisemblable qu'après la vente, les objets ne retourneront jamais au Pérou.

Le Pérou fait partie des Etats d'Amérique latine qui sont particulièrement affectés par les pillages de tombes (et les destructions qui les accompagnent toujours). Les Etats-Unis, qui ont également ratifié la Convention de I'UNESCO de 1970, ont pour cette raison conclu des accords bilatéraux complémentaires avec certains pays (Pérou, Guatemala, Mexique), qui prévoient la restitution de biens culturels exportés illégalement.

Source :

Site de la Déclaration de Berne : http ://www.access.ch/evb Facts 46/1995, "Heisse Ware unter dem Hammer "

${ }^{14}$ Dossier de presse du DFI, janvier 1996, « Commentaires sur la Convention de l'UNESCO de 1970 et la Convention d'Unidroit ». 
Partant de l'idée que seule une convention internationale peut espérer uniformiser tant soit peu les systèmes juridiques de quelque 200 Etats souverains, un comité d'experts présidé par le professeur Pierre A. Lalive d'Epinay de l'Université de Genève s'est attaché à la rédaction d'une Convention d'Unidroit sur les biens culturels. Le commerce de l'art étant principalement d'ordre privé (contrats de vente, transferts de propriété à un acquéreur de bonne foi, etc.) il devenait indispensable d'aborder ce problème sur le terrain du droit privé. L'objectif était d'éviter que les milieux intéressés tirent parti de la diversité des lois en vigueur dans les différents pays, tout en relayant efficacement les déclarations de principe qui fonde la Convention de l'UNESCO.

Les experts ont cependant rencontré un certain nombre de difficultés, sur la délimitation de la matière à réglementer et sur la définition de la notion de «biens culturels ", ainsi que sur la grande diversité des règles et des traditions juridiques nationales.

Comme le rappelle le Professeur Lalive d'Epinay ${ }^{15}$ "l'expansion continue du commerce illicite et en particulier du vol des biens culturels constitue un indéniable scandale, contre lequel il est urgent de lutter le plus efficacement, et qui porte préjudice aux particuliers comme aux collectivités, aux collectionneurs, aux musées, ainsi qu'aux objets culturels eux-mêmes (comme le montre l'exemple des fouilles archéologiques clandestines). » Pour contrer cette situation, la Convention s'est donné un double objet. Elle porte sur les biens culturels volés (chapitre II) et sur les biens culturels illicitement exportés (chapitre III), qui constitue un tout, à accepter ou à rejeter en bloc.

A la différence de la Convention de l'UNESCO, la Convention d'Unidroit est d'application directe (self-executing) ${ }^{16}$, c'est-à-dire qu'il n'est pas nécessaire d'intégrer ses dispositions dans la législation nationale des Etats contractants. C'est la première fois que sont énoncées, dans un acte d'importance générale, les conditions garantissant le retour ou la restitution des biens culturels volés ou exportés illicitement et réglant la procédure permettant de faire valoir des droits de restitution à l'échelon international. Deux points méritent d'être relevés ; premièrement, la Convention n'a pas d'effet rétroactif ; aussi ne s'applique-t-elle pas aux biens culturels qui ont été volés ou exportés illicitement avant son entrée en vigueur, deuxièmement, elle prévoit le paiement d'une indemnité équitable à tout possesseur de bonne foi d'un bien culturel volé ou illicitement exporté lorsqu'il a restitué le bien en question. Au deuxième chapitre de la Convention est énoncé un droit à la restitution des biens culturels volés ou issus de fouilles illicites, ainsi que les conditions requises pour la restitution des objets volés. Le troisième chapitre prévoit un droit au retour des biens illicitement exportés du territoire d'un Etat contractant, alors que la législation de ce dernier interdit de telles exportations. Un certain nombre de conditions doivent être remplies afin que les

\footnotetext{
${ }^{15}$ in « Une avancée du droit international : la Convention de Rome d'Unidroit sur les biens culturels volés ou illicitement exportés ", Revue de droit uniforme 1996-1, p. 48.

${ }^{16}$ Les traités « self-executing " s'appliquent directement dans chaque Etat contractant, sans adoption préalable de dispositions internes. Après leur ratification et leur publication, ils sont incorporés au droit national, ce qui signifie qu'ils se transforment automatiquement en source du droit, au même titre que la Constitution, la loi, l'arrêté de portée générale ou l'ordonnance. Le Conseil fédéral peut se fonder sur un traité « self-executing " pour édicter les prescriptions nécessaires à son exécution ; agissant par voie d'ordonnance, il se bornera à « concrétiser » les normes conventionnelles, sans en créer de nouvelles. Pour être « self-executing ", les clauses d'un traité doivent être suffisamment précises, c'està-dire aptes à servir de base à la solution de cas d'espèce ; (...) in André Grisel, Traité de droit administratif, vol. 1, éd. IDES et CALENDES, Neuchâtel, 1994, p. 91.
} 
biens culturels d'importance significative, exportés illicitement, puissent retourner dans les pays d'origine qui en font la demande.

La Convention d'Unidroit permet de lutter contre les pratiques illicites dans le commerce international de biens culturels. Parallèlement, elle met à la disposition de tout propriétaire légitime, qu'il s'agisse d'un Etat, d'un musée ou d'un collectionneur, un outil juridique lui donnant les moyens de faire valoir ses droits devant un tribunal ordinaire pour retrouver des biens culturels volés ou illicitement exportés. Par conséquent, si un possesseur ou un Etat étrangers faisaient valoir des droits de restitution, il reviendrait aux tribunaux et aux juges suisses de décider de la légitimité de ces droits.

En janvier 1996, le Conseil fédéral a autorisé le Département fédéral de l'intérieur à soumettre à une procédure de consultation la Convention d'Unidroit, invitant les milieux concernés à rendre leur avis sur sa ratification. Cette question soulève plusieurs domaines revêtant une importance capitale pour l'avenir de la politique suisse en matière de transfert international de biens culturels. Le nombre des avis rendus, plus de 120 , est considérable : cantons, partis politiques, organisations économiques et de commerçants d'art, ainsi que des organisations culturelles, de coopération au développement, etc. ont remis des commentaires denses et circonstanciés. Une très large majorité des participants à la procédure de consultation se sont déclarés favorables à la ratification de la Convention et ont approuvé les mécanismes qu'elle propose. Vingt-trois cantons, le PDC et le PS, l'Union syndicale suisse, les organisations intercommunales ou internationales, la très grande majorité des organisations culturelles, ainsi que les organisations ecclésiastiques, féminines et d'aide au développement, sont favorable à la ratification. Les cantons de Berne et de Genève, le PR et l'UDC, le Vorort et l'Usam, ainsi que les organisations de commerçants d'art, sont hostiles à la ratification de la Convention d'Unidroit.

Les partisans de la ratification saluent le fait que le Conseil fédéral souhaite prendre de nouvelles mesures rigoureuses destinées à lutter contre la progression inquiétante d'abus en matière de transfert international de biens culturels. A leurs yeux, la convention constitue un instrument très efficace pour combattre de tels abus. Dès lors, la ratification de la Convention d'Unidroit s'impose pour des raisons éthiques, juridiques et de politique culturelle. Sa non-ratification priverait la Suisse de mécanismes de protection efficaces et renforcerait nettement l'attrait qu'exerce notre pays sur les trafiquants d'art qui se livrent à des transactions douteuses. Les adversaires de la ratification estiment pour leur part que la Convention dépasse l'objectif visé et que les mécanismes qu'elle prévoit ne sont pas à même de tenir compte équitablement de tous les intérêts en jeu dans le transfert international de biens culturels. Ils craignent par ailleurs qu'elle n'entrave fortement le commerce d'art et l'activité des collectionneurs.

Le Conseil fédéral a pris connaissance des résultats de la consultation. Une très large majorité des participants s'étant déclaré favorable à la ratification de la Convention d'Unidroit ${ }^{17}$, il a décidé de la signer, signature déposée le 30 juin 1996 à Rome. Un groupe de travail sous la conduite du DFI a été institué pour examiner les questions juridiques en relation avec la convention de l'UNESCO de 1970 et la Convention d'Unidroit et coordonner la suite des travaux. ${ }^{17}$ Pour de plus amples détails, consulter les résultats de la procédure de consultation de la Convention d'Unidroit, à com-
mander au DFI. 


\section{Exemple de demande de restitution par des Etats Exportation illégale en provenance de la Turquie de stèles antiques}

Depuis 1979, cinq stèles phrygiennes, qui avaient été exportées illégalement de Turquie dans les années septante, sont exposées au Musée des arts antiques de Bâle. La Turquie a porté plainte dans le but d'obtenir le retour des ces stèles et a fait appel auprès du Tribunal fédéral suite à un jugement rendu par la Cour d'Appel de Bâle en 1995.

En 1973, dans le village turc de Gökceler, deux des stèles en question avaient été examinées, photographiées et dessinées par le professeur allemand Thomas Brew-Bear. Selon les déclarations des villageois, elles avaient été déterrées peu de temps auparavant par des indigènes. Le professeur Drew a tout de suite annoncé la trouvaille aux autorités du village. A la fin des années quatre-vingt, Drew-Bear a à nouveau reconnu les deux stèles sur des reproductions parues dans une étude scientifique. Une des stèles avait été sciée en deux pour des raisons commerciales. Après en avoir eu connaissance, les autorités turques ont réagi en déposant une demande de restitution en 1990.

La Cour d'Appel de Bâle a considéré le témoignage du Prof. Drew comme crédible. Malgré cela la plainte de la Turquie a été rejetée par la Cour d'Appel de Bâle, les exportations illégales n'étant pas reconnues comme des actes délictueux en Suisse. II fut reconnu que ce que l'on trouve sur le sol d'un Etat est bel et bien sa propriété, mais la Cour argumentât que les autorités turques avaient omis d'enregistrer rapidement les stèles. Etant donné que les stèles se trouvaient dans un petit village de campagne, cet argument fut remis en question. Le cas fut amené devant le Tribunal fédéral et l'arrêt du TF n'a, à ce jour, pas été rendu.

La Convention d'Unidroit n'est pas rétroactive. Si la Suisse l'avait signée en 1995, la situation juridique n'aurait pas changé pour cette plainte. Pour les exportations illégales, qui surviendraient après la ratification d'Unidroit, la Convention serait un instrument efficace pour garantir les droits des propriétaires légitimes.

Source:

Site de la Déclaration de Berne : http ://www.access.ch/evb

Basler Zeitung: 11.8.1995, "Türkei fordet von Basel antiche Grabsteine zurück », 19.8.1995, "Antike Grabsteine können in Basel bleiben ".

\section{CONCLUSION}

Le commerce des biens culturels volés et le trafic illicite sont en constante et inquiétante expansion. Pour faire face à cette situation, une collaboration internationale est indispensable. Collaboration à laquelle devraient prendre part les musées, les commerçants en œuvre d'art (en renforçant leur code de déontologie), l'administration, la police, les assureurs, les juristes, etc. Chacun s'accorde à reconnaître que ce problème ne peut être résolu sur le seul plan national. La rédaction de deux conventions internationales, à 25 ans d'intervalle, permet de croire que la plupart des nations reconnaissent aujourd'hui la nécessité de réduire les zones d'ombre dans la circulation internationale des œuvres d'art. De plus, compte tenu de la difficulté de nombreux Etats « exportateurs » à faire appliquer efficacement leur propre législation en la matière, la collaboration des pays « importateurs » se justifie par des exigences politiques et morales, propre à la solidarité internationale et à la protection des leurs propres biens culturels.

Comme nous l'avons démontré, le fossé se creuse entre le droit européen et l'ordre juridique helvétique. La Suisse, attractive pour de nombreux commer- 
çants, dont certains sont peu scrupuleux, apparait comme une plaque tournante du commerce illicite de l'art. Une série de scandales récents ne peut malheureusement que confirmer cette analyse. On ne peut tolérer plus longtemps que le territoire suisse serve ce commerce illégal et les résultats des deux consultations qui ont eu lieu récemment auprès des milieux intéressés laissent percevoir la volonté d'en finir avec cette situation inconfortable. Il est devenu urgent d'agir et le Conseil fédéral l'a bien compris puisque la Suisse a joué un rôle très actif dans les travaux préparatoires et lors de la conférence d'Unidroit à Rome et qu'elle a été parmi les premiers signataires de la Convention d'Unidroit. Certaines étapes importantes ont été franchies et des décisions politiques doivent maintenant confirmer ces avancées ; la ratification des Conventions de l'Unesco et d'Unidroit. Gageons que la Suisse sache prendre les mesures qui lui permettront de regagner sa crédibilité auprès de la communauté internationale.

\section{Chronologie des faits marquants}

Janvier 1990 Répondant aux appels de plus en plus pressants de l'étranger, et suite à plusieurs affaires survenues en Suisse, l'Office fédéral de la culture a constitué le 7 janvier 1990 un groupe de travail chargé d'étudier la question de l'exportation de biens culturels suisses et de l'importation de biens culturels étrangers et de formuler des propositions de solution.

Janvier 1991 Son rapport "Le commerce, l' exportation et l'importation de biens culturels en Suisse. Portée nationale et internationale; problèmes, solutions et effets » est remis en janvier 1991.

Le rapport propose principalement deux mesures :

- la ratification de la Convention de l'UNESCO de 1970 concernant les mesures à prendre pour interdire et empêcher l'importation, l'exportation et le transfert de propriété illicites des biens culturels ;

- la création d'une disposition constitutionnelle donnant à la Confédération compétence pour réglementer l'importation et l'exportation de biens culturels.

\section{Programme de législature 1991-1995}

Dans le rapport du 25 mars 1992 sur le Programme de la législature 19911995, le Conseil fédéral déclarait : «En matière de commerce des biens culturels, il existe également un besoin de réglementation. Dans ce secteur, 
la Suisse ne dispose pas, contrairement à la plupart des Etats européens, de lignes directrices réglant les possibilités de contrôle et de taxation. Le reproche fait à notre pays d'être le lieu de transit d'un commerce illégal de biens culturels étrangers n'est pas tout à fait infondé » (FF III 116). Le Conseil fédéral disait son intention d'aborder le problème du commerce de biens culturels et d'étudier les deux mesures proposées par le rapport du groupe de travail (FF 1992 III 117).

Avril 1992 Le rapport est rendu public plus d'une année après sa parution.

Parution du livre de la Déclaration de Berne "Götter, Gräber und Geschäfte, Von der Plünderung fremder Kulturen ", qui souligne notamment le rôle de plaque tournante de la Suisse dans le commerce de l'art.

1992-1993 Interventions parlementaires

- Motion de la conseillère nationale Grossenbacher du 18.6.1992

Cette motion constate que " la Suisse est une plaque tournante importante du trafic d'œuvres d'art et de biens culturels ». Elle demande au Conseil fédéral de prendre des dispositions législatives pour empêcher ce trafic, de ratifier la Convention de l'UNESCO de 1970, d'édicter une loi d'introduction correspondante, d'encourager les cantons à lancer des campagnes d'information et d'harmoniser le droit suisse sur celui de la CE.

Le Conseil national a accepté la motion le 25 juin 1993. Elle va maintenant être examinée par le Conseil des Etats.

- Postulats des commissions de la science, de l'éducation et de la culture du Conseil national et du Conseil des Etats, 1993

Les commissions de la science, de l'éducation et de la culture du Conseil national (22.1.1993) et du Conseil des Etats (4.5.1993) ont toutes deux déposé un postulat de même teneur exhortant le Conseil fédéral à signer sans délai la Convention de l'UNESCO de 1970 et à édicter des dispositions législatives propres à éviter l'exportation des biens nationaux et à soutenir les pays étrangers dans leurs efforts visant à sauvegarder leur patrimoine culturel.

Ces postulats ont été transmis l'un à la session de printemps, l'autre à la session d'été 1993.

- Motion du conseiller national Keller du 4. 3.1993

Cette intervention concerne un aspect particulier du transit des biens culturels : il demande au Conseil fédéral de soutenir les efforts entrepris au plan national et international pour regrouper les biens culturels. La motion a été transmise sous forme d'un postulat à la session d'été 1993.

Septembre 1993 Procédure de consultation pour la ratification de la Convention de l'UNESCO de 1970 et concernant l'établissement d'une compétence habilitant la Confédération à légiférer en matière de transfert international de biens culturels.

Janvier 1996 Les résultats de la procédure de consultation lancée en septembre 1993 sont rendus publics.

Janvier 1996 Procédure de consultation concernant la Convention d'Unidroit du 24 juin 1995 sur les biens culturels volés ou illicitement exportés.

Mai 1996 Les résultats de la procédure de consultation lancée en janvier 1996 sont rendus publics.

30 juin 1996 Signature de la Convention d'Unidroit par la Suisse (décision du Conseil fédéral). 


\section{SOURCES}

Documents officiels :

Convention du l'UNESCO de 1970 concernant les mesures à prendre pour interdire et empêcher l'importation, l'exportation et le transfert de propriété illicites de biens culturels

Convention d'Unidroit du 24 juin 1995 sur le retour international des biens culturels volés ou illicitement exportés.

Rapport du groupe de travail à l'intention de l'Office fédéral de la culture et du Département fédéral de l'intérieur, « Le commerce, l'exportation et l'importation de biens culturels en Suisse. Portée nationale et internationale ; problèmes, solutions et effets ». Janvier 1991

Texte et rapport explicatif du Département fédéral de l'intérieur, « Commerce, importation et exportation de biens culturels ", Berne, août 1993

Texte et rapport explicatif du Département fédéral de l'intérieur, « Convention Unidroit », février 1996

Résultats de la procédure de consultation portant sur « Le commerce de biens culturels ", Office fédéral de la culture, 1995, ainsi que le communiqué de presse relatif (DFI, janvier 1996)

Résultats de la procédure de consultation portant sur « La convention d'Unidroit », Office fédéral de la culture, mai 1996, ainsi que le communiqué de presse relatif (DFI, juin 1996)

\section{Publications :}

La libre circulation des collections d'objet d'art, Quentin Byrne-Sutter et Marc-André Renold, Centre du droit de l'art, éd. Stämpfli, Zurich, 1993

Revue de droit uniforme 1996-1, «Une avancée du droit international : la Convention de Rome d'Unidroit sur les biens culturels volés ou illicitement exportés », Pierre Lalive d'Epinay

Götter, Gräber und Geschäfte, Von der Plünderung fremder Kulturen, Elisa Fuchs, Déclaration de Berne, Avril 1992 (disponible en allemand uniquement)

\section{Presse :}

EvB Dokumentation, 2/1992, "Geplünderte Kulturen - grosse Geschäfte »

Vers un développement solidaire, « Le marché de l'art ", $\mathrm{n}^{\circ} 113$, juillet 1992

Le Courrier, 19 février 1994, "La liberté du commerce de l'art suscite une très vive controverse "

La Liberté, 18 juillet 1995, «La restitution d'œuvres d'art volées sera réglementée "

Journal de Genève ; 26 juin 1995, Accord sur la restitution d'œuvres d'art volées, 20 novembre 1995, « Pour les marchands d'art, la Suisse est un ilôt de liberté qu'ils comptent bien protéger », 18 janvier 1996, « La Suisse aimerait mieux protéger les biens culturels étrangers », 3 mai 1996, supplément Le marché de l'art, 7 juin 1996, « Réglementer le marché de l'art, le Conseil fédéral doit bientôt trancher»

Neue Zürcher Zeitung, 14 janvier 1994, « Umstritte Handelsvorschriften für Kulturgüter », 18 janvier 1996, « Dubiosen Geschäften mit Kulturgütern einen Riegel schieben », 15 juin 1996, « Unidroit - Kulturgüterrecht auf Irrwegen », 19 juin 1996, « Unterzeichnung des Kulturgüterschutzkonvention »

Enregistrement de l'émission Forum de la radio suisse romande, 16 mai 1994, Le marché de l'art en Suisse

\section{SITE INTERNET :}

http ://www.access.ch/evb

Dossier sur le thème de la Déclaration de Berne, en allemand. Der illegale Kulturgüterhandel und die Rolle der Schweiz

\section{ADRESSE UTILE :}

Centre de droit de l'art, Case postale 176, 1211 Genève 12 